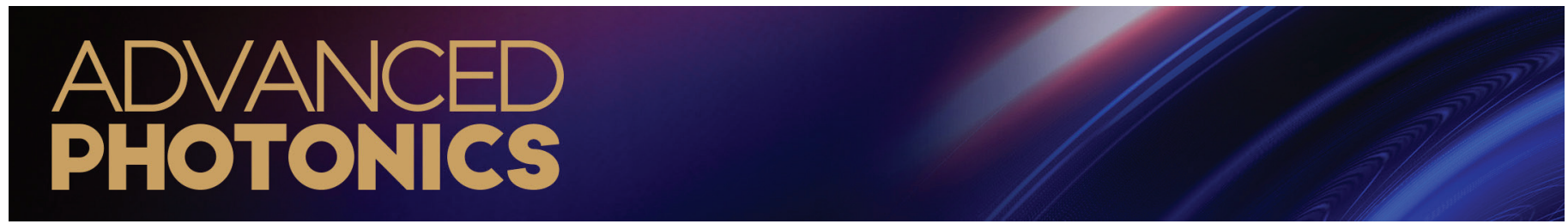

\title{
Robust and rapidly tunable light source for SRS/CARS microscopy with low-intensity noise
}

\author{
Heiko Linnenbank, ${ }^{\mathrm{a}, \mathrm{b}, \star}$ Tobias Steinle, ${ }^{\mathrm{a}, \mathrm{b}}$ Florian Mörz, ${ }^{\mathrm{a}, \mathrm{b}}$ Moritz Flöss, ${ }^{\mathrm{b}}$ Han Cui, ${ }^{\mathrm{c}}$ Andrew Glidle, ${ }^{\mathrm{c}}$ and \\ Harald Giessen ${ }^{\mathrm{a}, \mathrm{b}}$ \\ ${ }^{a} S I$ Stuttgart Instruments $\mathrm{GmbH}$, Öhringen, Germany \\ bUniversity of Stuttgart, 4th Physics Institute and Research Center SCoPE, Stuttgart, Germany \\ 'University of Glasgow, College of Science and Engineering, Division of Biomedical Engineering, Glasgow, United Kingdom
}

\begin{abstract}
We present a fully automated laser system with low-intensity noise for coherent Raman scattering microscopy. The robust two-color system is pumped by a solid-state oscillator, which provides Stokes pulses fixed at $1043 \mathrm{~nm}$. The tunable pump pulses of 750 to $950 \mathrm{~nm}$ are generated by a frequency-doubled fiberfeedback femtosecond optical parametric oscillator. The resulting pulse duration of 1.2 ps provides a viable compromise between optimal coherent Raman scattering signal and the necessary spectral resolution. Thus a spectral range of 1015 to $3695 \mathrm{~cm}^{-1}$ with spectral resolution of $<13 \mathrm{~cm}^{-1}$ can be addressed.
\end{abstract}

Keywords: label-free imaging; stimulated Raman scattering; optical parametric amplifier; spectral compression.

Received Jul. 4, 2019; accepted for publication Aug. 28, 2019; published online Sep. 24, 2019.

(C) The Authors. Published by SPIE and CLP under a Creative Commons Attribution 4.0 Unported License. Distribution or reproduction of this work in whole or in part requires full attribution of the original publication, including its DOI.

[DOI: 10.1117/1.AP.1.5.055001]

\section{Introduction}

Coherent Raman scattering (CRS) imaging is based on a multiphoton scattering process that employs two near-infrared laser pulses to excite Raman modes in the midinfrared spectral range. Owing to its chemical selectivity without labeling, it has found a wide scope of applications in biomedical microscopy, such as live cell, tissue, or DNA imaging, ${ }^{1-5}$ over the past years. Its most prominent representatives are coherent anti-Stokes Raman scattering (CARS) and stimulated Raman scattering (SRS). ${ }^{6}$ In the case of CARS, two beams, the so-called pump and Stokes beam, with an energy difference matching the Raman resonance $\Omega$, interact according to a four-wave-mixing process. Thus coherent radiation at the anti-Stokes frequency $\omega_{\mathrm{CARS}}=2 \omega_{P}-\omega_{S}$ is generated, as depicted in Fig. 1. In contrast to CARS, SRS is an inelastic scattering process, where energy is transferred from the pump to the Stokes beam, thus either the change in pump [stimulated Raman loss (SRL)] or Stokes intensity [stimulated Raman gain (SRG)] can be detected. SRS, unlike spontaneous spectroscopy and CARS, ${ }^{7}$ is therefore free from fluorescent and nonresonant background. In addition, SRS provides quantitative information about the chemical constituents, due to its linear signal scaling. ${ }^{8-10}$

*Address all correspondence to Heiko Linnenbank, E-mail: h.linnenbank@pi4.unistuttgart.de
Although in the case of CARS a very weak but spectrally easy to separate third beam is generated, a tiny change of either the pump or the Stokes beam has to be measured for SRS. Thus lock-in-based detection is required for the latter. ${ }^{1}$ This implies that for SRS an ultralow-intensity laser noise, ideally at the shot-noise limit, is required. Simultaneously, excellent longterm average power stability and reproducibility are required for laser scanning imaging, and at least one color must be rapidly tunable while maintaining its spatiotemporal properties. The main regions of interest are the H-stretching vibrational region (2500 to $3500 \mathrm{~cm}^{-1}$ ) as well as the fingerprint region (500 to $1700 \mathrm{~cm}^{-1}$ ). In order to face real-world applications, the source is required to be cost-effective and easy to use, and thus to be fully automated.

The vast majority of work in CRS in the past decade was based on picosecond, green-pumped optical parametric oscillators (OPOs), ${ }^{8,11}$ as well as electronically synchronized Ti:sapphire laser systems. ${ }^{9}$ Recent demonstrations of novel CRS sources include Fourier-transform spectral retrieval, ${ }^{12}$ chirped pulse spectral focusing, ${ }^{13,14}$ as well as photonic time stretch, ${ }^{15}$ ps-laser diodes, ${ }^{16}$ and swept-source SRS. ${ }^{17}$ These techniques are able to achieve higher information volume ${ }^{6}$ but to date are still quite complex to operate and exhibit alignment sensitivity of the detection system. 
(a)

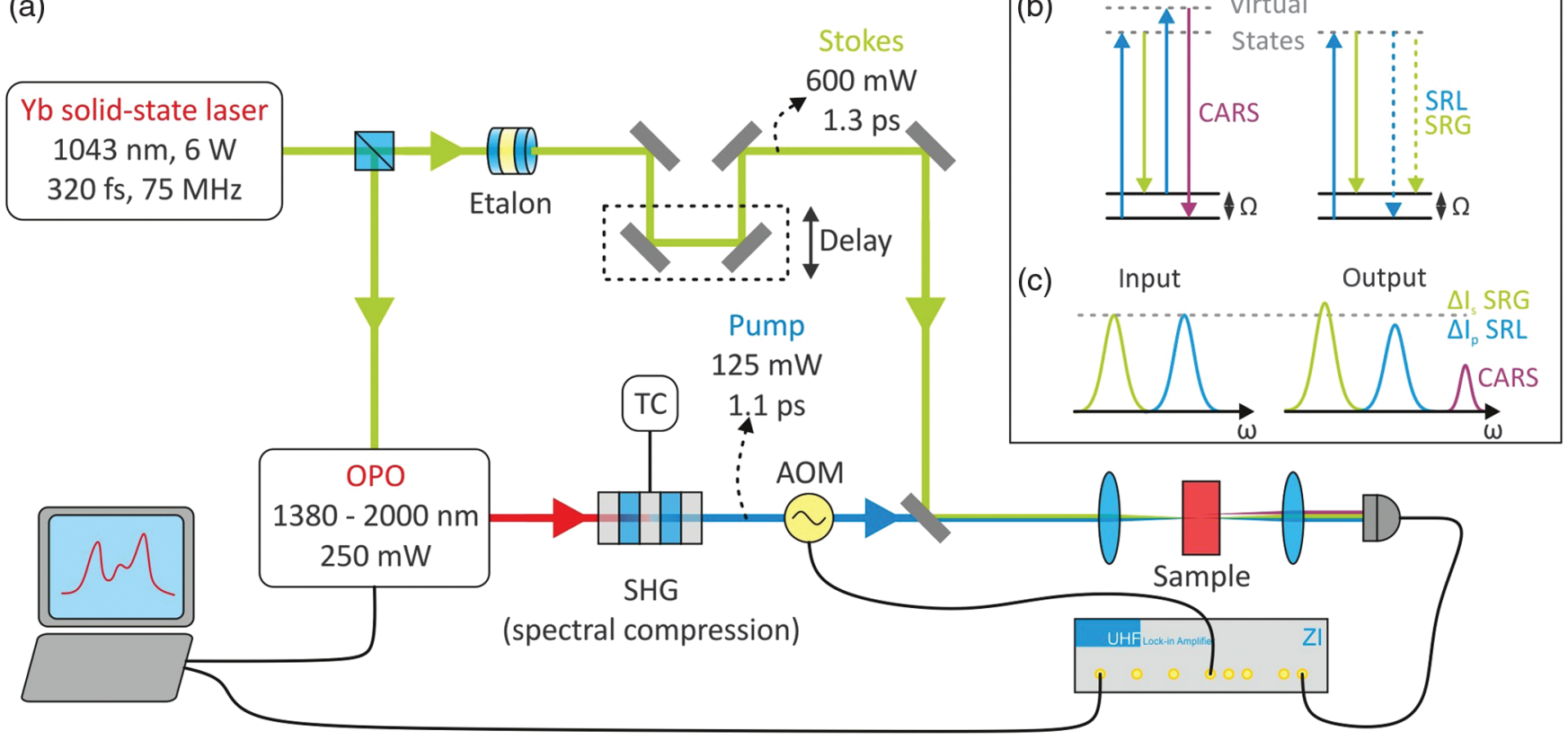

Fig. 1 (a) Schematic experimental setup. An Yb-solid-state oscillator allows synchronous generation of the Raman Stokes and pump beams. Using an etalon, the narrowband Stokes beam with picosecond pulse duration is obtained, whereas the Raman pump beam is generated by pumping an OPO, which is subsequently frequency doubled in a periodically poled crystal employing the effect of spectral compression. Using this system, CARS and SRS spectroscopies are both feasible, as depicted in (b) and (c).

Although avoiding the aforementioned complexity, fiber lasers possess high-relative intensity noise in the $\mathrm{MHz}$ regime $^{18-20}$ due to amplified spontaneous emission in the rather long gain region of the fiber. Balanced detection is typically used to overcome this issue, at the cost of significant complication of the detection system to handle spatially variable transmission of scattering specimens. Last but not least, fiber laser and amplifier technology is limited to certain spectral ranges in the near-infrared around $1050 \mathrm{~nm}(\mathrm{Yb}), 1540 \mathrm{~nm}$ $(\mathrm{Er})$, and $1880 \mathrm{~nm}(\mathrm{Tm})$, thus also limiting the accessible Raman bands from a single system.

Here we present a CRS source that combines a broad tuning range, a low-intensity noise, and rapid broadband tunability. Our approach employs the high efficiency and broad tunability of a fiber-feedback OPO operating in the few hundred femtoseconds pulse regime at several tens of MHz. Pumped by a solid-state $\mathrm{Yb}$-oscillator those devices exhibit excellent stability on all timescales. ${ }^{21,22}$ To achieve the desired spectral resolution as well as the desired frequency range for CRS, we employ spectral compression second-harmonic generation $(\mathrm{SHG}) .^{23}$ This allows us to cover a spectral range of 1015 to $3695 \mathrm{~cm}^{-1}$ with spectral resolution of below $13 \mathrm{~cm}^{-1}$ and average powers above $50 \mathrm{~mW}$ for the pump and $600 \mathrm{~mW}$ for the Stokes beam.

\section{Optical Setup}

As depicted in Fig. 1(a), our CRS system is based on an Ybsolid-state oscillator, which provides 6-W average output power at $1043-\mathrm{nm}$ central wavelength. About 390-fs pulses are measured at a pulse repetition rate of $75 \mathrm{MHz}$. A $2.5-\mathrm{W}$ fraction of this oscillator is then used to pump a fiber-feedback OPO, in which tunable infrared light between 1380 and $2000 \mathrm{~nm}$ is generated..$^{21,22}$ In such a device, the synchronized optical cavity is divided in a short-free space part, constituted by the nonlinear optical crystal and its focusing optics, and a long single-mode fiber. Using a single-mode fiber for the optical feedback, the optical path length of $4 \mathrm{~m}$, given by the pump laser repetition rate, can be implemented in a very compact setup. As the freespace part is minimized to a length of few tens of centimeters, it becomes less sensitive to misalignment compared to entire free space cavities. Furthermore, the optical fiber intrinsically acts as a mode filter and determines the pointing. ${ }^{21,22}$ The OPO is passively stable and does not require an active feedback loop, thus avoiding any kind of additional noise or power fluctuations, which could deter the coherent Raman images. Also the fiber feedback technology allows one to employ alternative $\mathrm{Yb}$-femtosecond lasers in the range of 10 to $100 \mathrm{MHz}$. To generate the Raman pump beam, this infrared light is frequency doubled and temporally stretched to about 1.1-ps pulse duration by spectral compression SHG in a periodically poled lithium niobite crystal with a length of $12.5 \mathrm{~mm}$ to tailor the bandwidth. ${ }^{23}$ To achieve a fast accessible, broad tuning range, the crystal consists of 19 different poling periods and its temperature is adjustable. An air-spaced etalon is used to spectrally narrow the pulses of the remaining fraction of the Yb-solid-state oscillator beam, leading to a Raman Stokes beam with an average power of $600 \mathrm{~mW}$ and a pulse duration of 1.3 ps. The Raman pump and Stokes beams are spatially combined using a dichroic mirror. To ensure temporal synchronization, a delay stage is inserted into the Stokes beam with a mechanical resolution of $1.5 \mu \mathrm{m}$, which translates to a temporal delay of $<5$ fs. To modulate the Raman pump beam at external triggerable frequencies of 
up to $20 \mathrm{MHz}$, the beam is tightly focused into an AOM. In order to prevent a change of the beam pointing due to wavelengths changes, the 0'th order diffraction is employed, which provides a modulation depth of $50 \%$ to $90 \%$ depending on the wavelength. The AOM could in principle also be used for the Stokes beam to operate the setup in SRL configuration.

This system is fully automated and can be controlled specifically for a CRS designed controller or via a computer interface. The entire tuning range is accessible within $20 \mathrm{~s}$ by a single command in steps of $9 \mathrm{~nm}$. The step width is a result of the phase-matching arising from the different poling periods of the crystal. If fine tuning is required, the pump wavelength can be adjusted by temperature tuning with subnanometer precision. The temperature adjustment takes $<1 \mathrm{~min}$. The wavelength shift with respect to the crystal temperature reads only $0.08 \mathrm{~nm} / \mathrm{K}$. Thus the wavelength is very precisely and reproducibly determined by the crystal temperature and the poling period. This allows one to implement the wavelength control via a simple look-up table without the need for a subsequent spectral analysis. Due to the high-average pump and Stokes power, this system is suited for both CARS and SRS. For validation experiments, a simple confocal setup is employed, which will be described later.

\section{System Performance}

In Fig. 2(a), the near-infrared signal output spectra of the OPO are depicted along with the corresponding output powers. Although not depicted in Fig. 1, these near-IR wavelengths are available via a second output port in the featured device and usable for applications such as multiphoton fluorescence microscopy. The signal can be tuned between 1.38 and $2.02 \mu \mathrm{m}$ with a full width at half maximum (FWHM) of the individual spectra of 9 to $20 \mathrm{~nm}$, with nearly constant pulse durations of 200 to $300 \mathrm{fs}$. Up to $500 \mathrm{~mW}$ is generated at $1600 \mathrm{~nm}$ and more than $200 \mathrm{~mW}$ power is achievable between 1.38 and $1.86 \mu \mathrm{m}$. All spectra exhibit minor sidelobes, which exhibit an amplitude of $<15 \%$ of the peak intensity.

In Figs. 2(b) and 2(c), the combined pump and Stokes output spectra are presented. The spectrum of the filtered Yb-laser used as Stokes beam in CRS experiments is depicted in Fig. 2(c) together with the unfiltered spectrum (dashed line). The filtering leads to a maximum Stokes power of $600 \mathrm{~mW}$ at $1043.1 \mathrm{~nm}$, with FWHM bandwidth of $0.71 \mathrm{~nm}$, which corresponds to a resolution for the CRS measurements of $6.5 \mathrm{~cm}^{-1}$. As expected, the etalon provides a perfect Lorentzian lineshape. The entire tuning range, realized by exploiting spectral compression SHG, is presented in Fig. 2(b), together with a representative spectrum at $788 \mathrm{~nm}$ in Fig. 2(d), where in our case only a rough tuning grid has been used. From 753 up to 943 nm, more than $50 \mathrm{~mW}$ average output power can be achieved with a maximum level of $245 \mathrm{~mW}$. The FWHM bandwidth is linearly increasing with wavelength from 0.5 to $1.2 \mathrm{~nm}$; taking the medial value into account, a spectral resolution for CRS of better than $13.5 \mathrm{~cm}^{-1}$ is obtained. Again, small sidelobes reaching only $15 \%$ intensity of the main peak are visible. The measured conversion efficiency reaches $33 \%$ to $50 \%$.

The pulses of the pump beam are very close to the Fourierlimit, thus featuring durations below $1.4 \mathrm{ps}$. As visible in Fig. 2(d), the spectra of the pulses exhibit a sinc ${ }^{2}$ shape resulting from the employed phase matching. ${ }^{23}$ The resulting pulses (a)

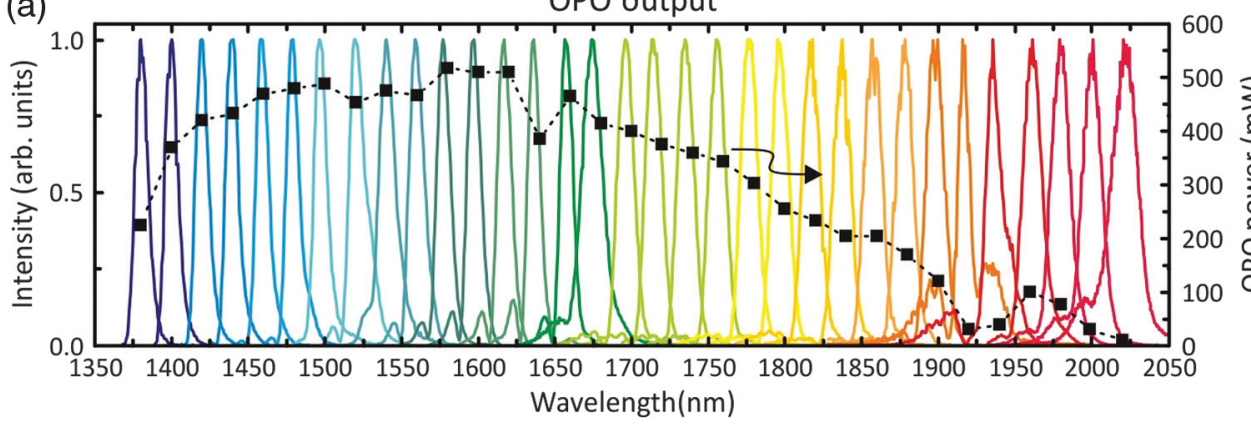

(b)

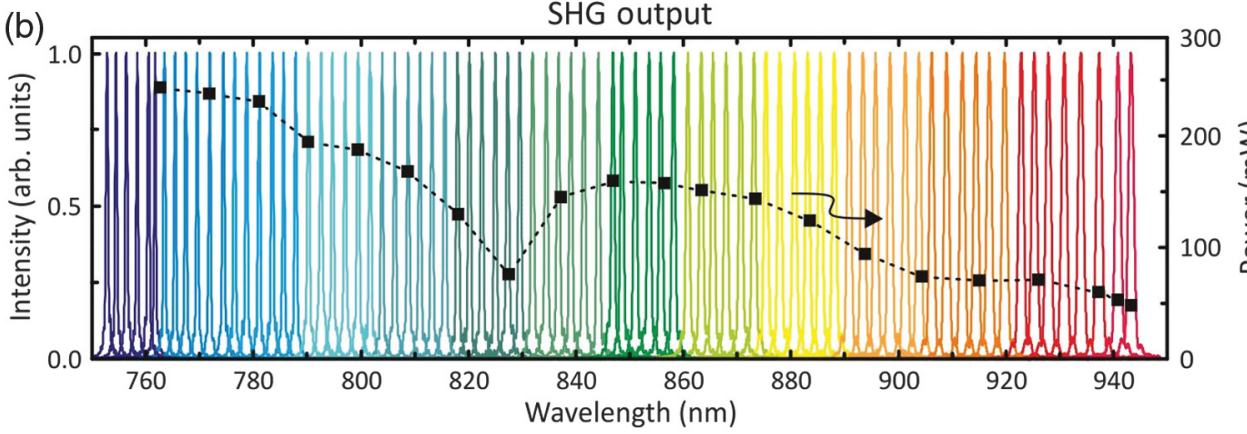

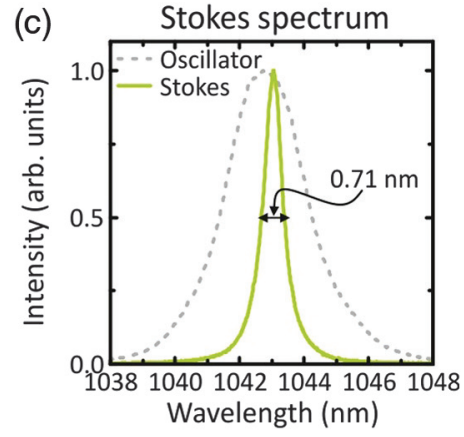

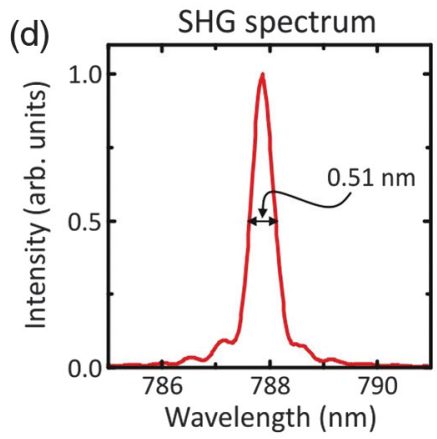

Fig. 2 (a), (b) Spectral range and typical output power of the broadband Raman laser system. (c) By employing an etalon, the Stokes beam reaches a bandwidth of $0.71 \mathrm{~nm}$ at $1043.1 \mathrm{~nm}$. The original solid-state oscillator spectrum is indicated in gray. (d) Typical spectrum within the Raman pump spectral range. Due to spectral compression during the SHG, the spectrum typically reaches a bandwidth between 0.5 and $1.2 \mathrm{~nm}$. 
should exhibit a rectangular pulse-shape, which in turn leads to a triangular autocorrelation, as visible in Fig. 3(a). The Stokes pulses displayed in Fig. 3(b), follow their spectral form leading to a Fourier-limited pulse duration of $1.26 \mathrm{ps}$. As coherent Raman scattering is a nonlinear process, the high-intensity resulting from close to Fourier-limited pulses is in fact preferable. Both spectral engineering techniques, the etalon filtering and the spectral compression SHG are of course adaptable to obtain a higher spectral resolution with longer and thus less intense pulses or the other way around if required for specific investigations.

The stable performance is demonstrated by recording the free running temporal output power traces of pump (SHG), OPO, and Stokes beams [see Fig. 4(c)] with a thermal power meter having a response time of $0.1 \mathrm{~s}$, revealing an excellent stability below $0.5 \% \mathrm{rms}$. The Stokes beam exhibits the highest stability, which is most likely due to the spectral filtering, as comparable measurements of the $\mathrm{Yb}$-oscillator itself (not shown) revealed a two times higher noise level.

Comparable results can be deduced from relative intensity noise measurements presented in Fig. 3(d), where also the unfiltered $\mathrm{Yb}$-oscillator is investigated. For all measurements, the optical intensities were adjusted such that a photocurrent of $1.5 \mathrm{~mA}$ was detected. Between 5 and $50 \mathrm{kHz}$, the OPO and the SHG show a similar noise level, which is 3 to $5 \mathrm{~dB}$ higher than that of the pump laser. In this frequency range, the stabilizing effect of the spectral filtering is even more obvious as the Stokes beam shows a more than $10 \mathrm{~dB}$ lower noise level than the unfiltered $\mathrm{Yb}$-oscillator. The origin of the narrow spikes visible at $4.6 \mathrm{kHz}$ and higher harmonics of that frequency in all beams are the driver electronics of the Yb-oscillator. Between 50 and $800 \mathrm{kHz}$, the noise level of all beams is decreasing, finally reaching the calculated electronic shot-noise level for a photocurrent of $1.5 \mathrm{~mA}$ within $2 \mathrm{~dB}$. Again, the Stokes beam exhibits a superior performance as shot-noise limited operation is already achieved at $200 \mathrm{kHz}$. The small spikes between 1 and $20 \mathrm{MHz}$ are an artefact of the detection electronics.

\section{SRS Measurements}

In order to evaluate the system performance, i.e., the tuning width and resolution, we recorded the SRS response from a 1:1 volume mixture of $\mathrm{D}_{2} \mathrm{O}$ and $\mathrm{H}_{2} \mathrm{O}$ by spectrally scanning with the same steps as in Fig. 2(b). Average powers of $30 \mathrm{~mW}$ have been employed for the pump beam modulated

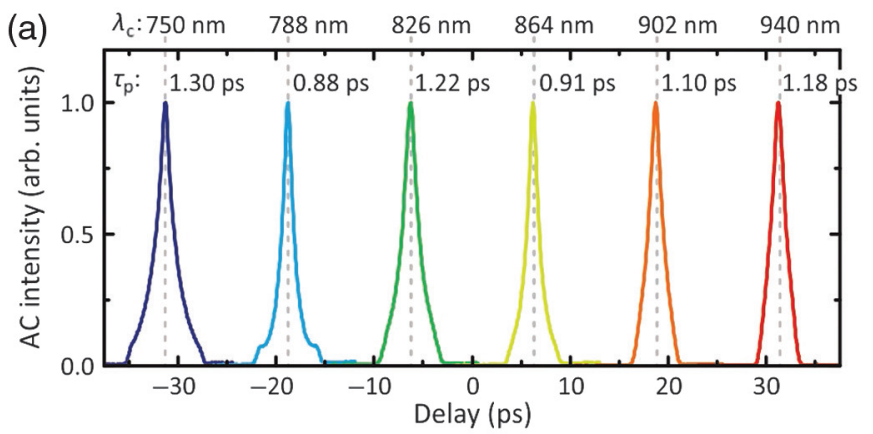

(b)
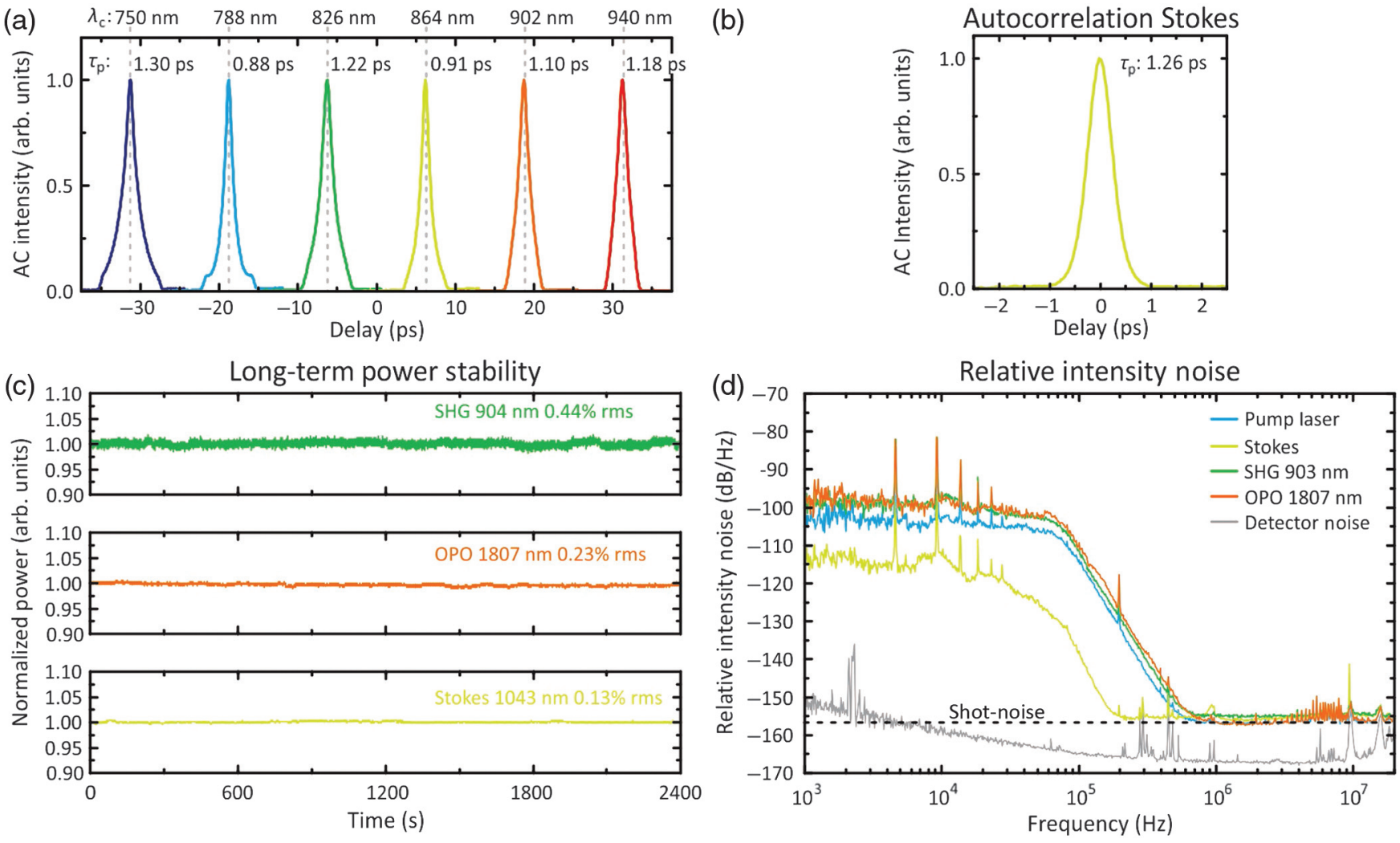

Fig. 3 (a) Autocorrelation traces of the Raman pump beam between 750 and $940 \mathrm{~nm}$. Nearly Fourier-limited pulses are observed over the entire Raman pump tuning range, exhibiting typical pulse durations of about 1.1 ps. (b) Autocorrelation of the Stokes beam. (c) Long-term power stability of the Stokes, OPO, and Raman pump (SHG) beams. Fluctuations below $0.5 \%$ rms are observed within the Raman pump spectral range, achieving excellent long-term stability. (d) Corresponding relative intensity noise measurements. The Stokes beam reaches the shotnoise limit at a photocurrent of $1.5 \mathrm{~mA}$ at about $150 \mathrm{kHz}$, whereas the OPO and the SHG (Raman pump) exhibit slightly higher noise. All beams are shot-noise limited above $1 \mathrm{MHz}$, enabling low-noise coherent Raman spectroscopy. 

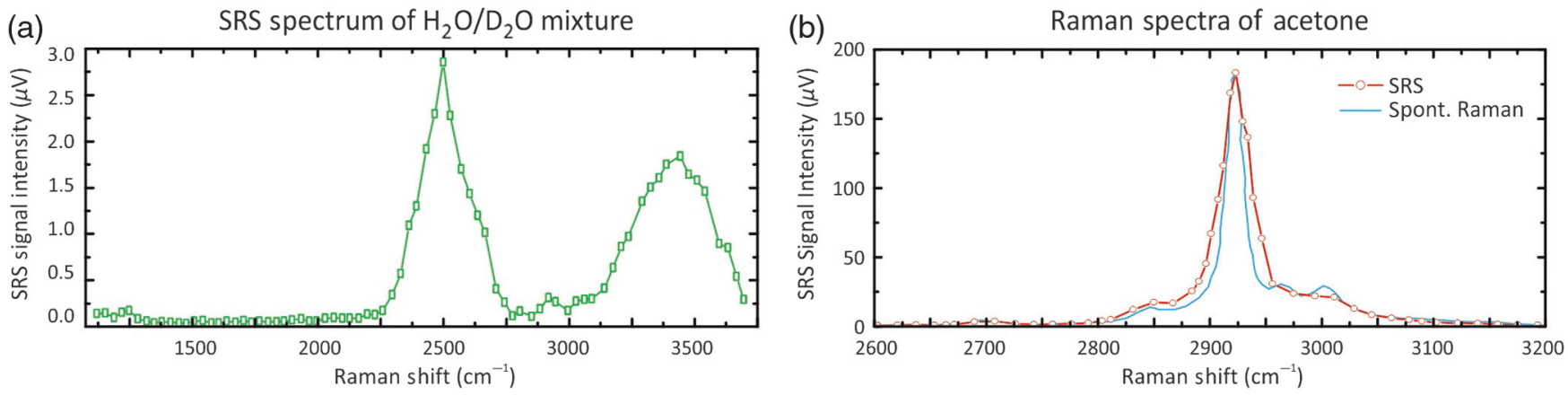

Fig. 4 (a) SRS spectrum of a 1:1 volume mixture of $\mathrm{D}_{2} \mathrm{O}$ and $\mathrm{H}_{2} \mathrm{O}$ recorded by employing the whole tuning range of the presented system. (b) SRG spectrum of acetone showing the characteristic resonance at $2923 \mathrm{~cm}^{-1}$. A spontaneous Raman spectrum is overlaid for comparison. The individual measurement points are connected as guide to the eye.

at $200 \mathrm{kHz}$. The beams have been focused to a diameter of $\sim 15 \mu \mathrm{m}$ in a cuvette with the help of aspheric lenses. For detection, a standard single-channel Si-photodiode (Thorlabs PDA100A2) has been employed. The depicted SRS signal intensity values are given by the demodulated signal voltages. Compared to the average detected signal of $3 \mathrm{~V}$, this means that relative signals below $10^{-6}$ have been resolved. The resulting spectrum clearly resolves the stretching vibrations of $\mathrm{OH}$ and $\mathrm{OD}$ at 3425 and $2500 \mathrm{~cm}^{-1}$. From spontaneous Raman measurements, ${ }^{24}$ it is known that both bands should exhibit weak shoulders at $3650 / 3250$ and 2690/2395 $\mathrm{cm}^{-1}$, which can already be discerned from this spectrally coarse scan. Finally, the ratio of the Raman signal between $\mathrm{OD}$ and $\mathrm{OH}$ band matches the expectations from the literature. ${ }^{24}$

To judge the accuracy of the retrievable Raman response, the spectrum of pure acetone has been measured and is compared to a spontaneous Raman signal in Fig. 4(b). Here average powers of $75 \mathrm{~mW}$ have been employed for the pump beam. The SRS spectrum clearly resolves the $\mathrm{CH}$-stretch band in acetone at $2923 \mathrm{~cm}^{-1}$. Compared to the spontaneous Raman spectrum, a slightly broader lineshape is obtained, which is however, in good agreement with the spectral resolution of below $13 \mathrm{~cm}^{-1}$ discussed in the previous paragraph. Nevertheless, all spectral features are clearly visible, and the relative strength of the shoulders compared to the main peak is exactly resolved.
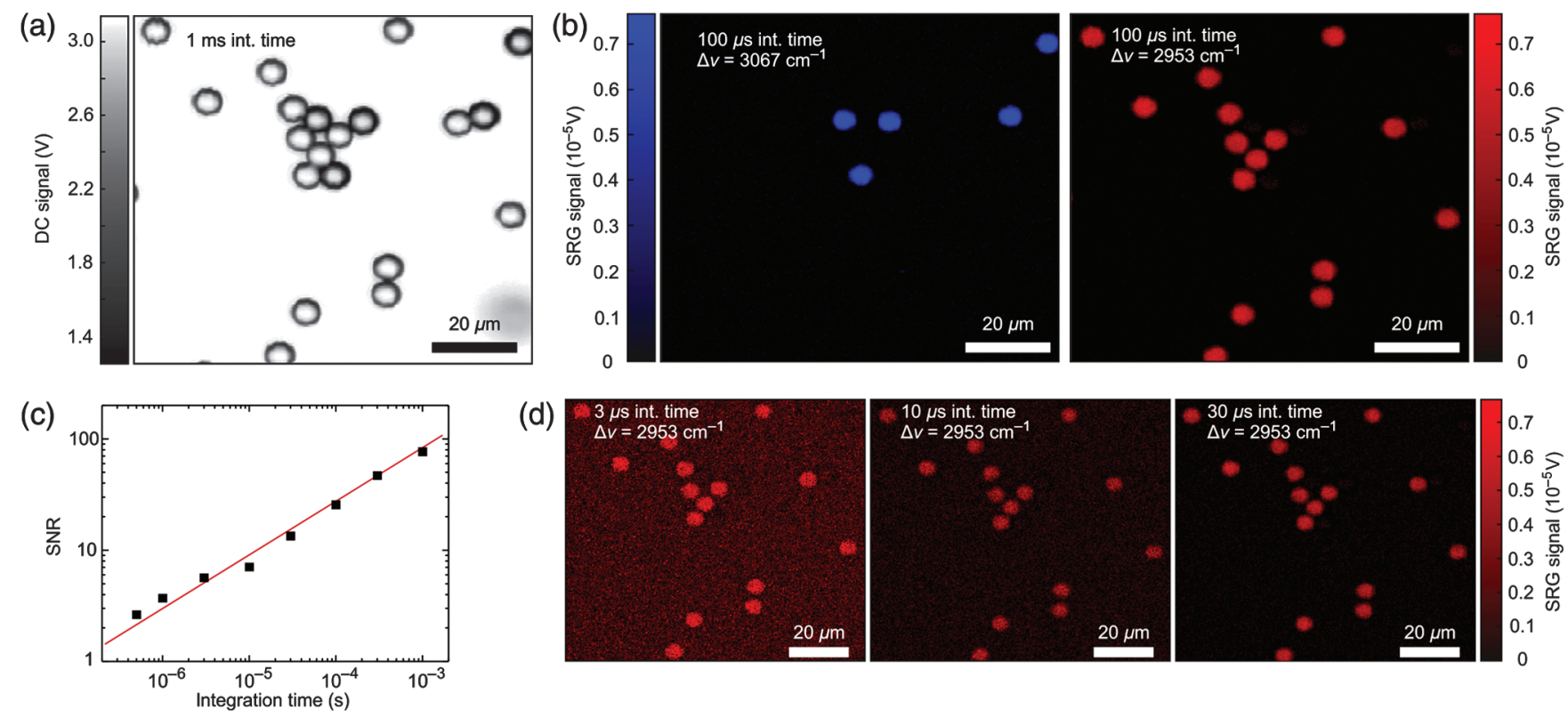

(d)
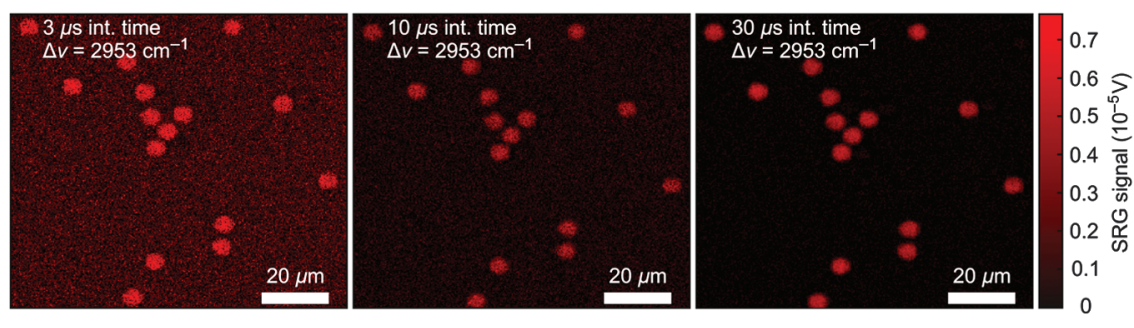

Fig. 5 (a) $94 \mu \mathrm{m} \times 94 \mu \mathrm{m}$ raster scan image of $6-\mu \mathrm{m}$ diameter polymer beads. The contrast mechanism is the scattering by the beads, which is lowering the DC-value of the detected Stokes beam. (b) SRG signal images from the raster scan in (a), recorded for a Stokes-pump energy difference of $3067 \mathrm{~cm}^{-1}$ (PS-band) and $2953 \mathrm{~cm}^{-1}$ (PMMA-band), revealing two different kinds of beads. (c) Signal-to-noise ratio extracted from images taken at $2953 \mathrm{~cm}^{-1}$ as a function of the pixel integration time. (d) SRG signal images recorded at $2953 \mathrm{~cm}^{-1}$ with ascending pixel integration times. 
To demonstrate the capability of the presented light source with respect to imaging applications, we employed a basic confocal scanning microscope setup consisting of just two objectives with a numerical aperture of 0.7 and a 2-D stage with a step width of $0.3125 \mu \mathrm{m}$. The detection is again performed with a standard single-channel Si-photodiode and a modulation of $1 \mathrm{MHz}$. As test sample, we investigated polymer beads with a diameter of 6- $\mu \mathrm{m}$ spread on a microscope coverslip. To avoid laser damage of those beads, the total incident optical power was kept at $15 \mathrm{~mW}$. By recording the DC value of the detected Stokes beam, we can construct a conventional laser raster scan image as shown in Fig. 5(a). Here the beads appear as dark rings due to the pronounced scattering at the edges. Recording the demodulated signal for the very same field of view with a Stokes-pump energy difference of $3067 \mathrm{~cm}^{-1}$, we can only find a few of the beads [see left panel of Fig. 5(b)]. This time they appear as intense spots on a dark background, which clearly identifies them as polystyrene (PS), as this exhibits a strong Raman mode at this particular frequency. ${ }^{25}$ By tuning to $2953 \mathrm{~cm}^{-1}$, the PS beads become nearly invisible, whereas the other beads appear again, unmasking them as polymethylmethacrylate (PMMA). ${ }^{25}$ In Fig. 5(c), the signal-to-noise ratio achieved for different pixel integration times is evaluated, revealing that detection with $<1-\mu$ s pixel integration time is possible. This becomes likewise evident by an inspection of the underlying images shown in Fig. 5(d). With more sophisticated microscope setups employing galvo mirrors, $300 \times$ 300 pixel images, as presented in Fig. 5(d), could thus be recorded within $<270 \mathrm{~ms}$, which means that video frame rate imaging is possible.

\section{Conclusion}

In this letter, we present a fully automated, user-friendly coherent Raman excitation light source, based on optical parametric frequency conversion of a solid-state oscillator. Using this system, resonances between 1015 and $3695 \mathrm{~cm}^{-1}$ can be detected at a spectral resolution of at least $13 \mathrm{~cm}^{-1}$. Due to the robust fiber-feedback design, no active stabilization electronics are required, which usually affect the system stability, noise, and handling in a negative manner. Owing to the advanced mechanical setup, this CRS system is insensitive regarding ambient conditions and thermal changes. Furthermore, the Stokes and pump beams are shot-noise limited well below $1 \mathrm{MHz}$, which enables low-noise Raman spectroscopy. Thus no sophisticated detection techniques, such as balanced heterodyning are required, and a standard single-channel silicon photodiode is applied to detect SRS signals. Our system is fully automated and allows wavelength tuning with subnanometer precision via its hardware control panel or by remote access. We believe that our system significantly simplifies the entrance of nonexperts in optics and laser technology to stimulated Raman microscopy and spectroscopy, bringing this technique closer to biologists and medical experts and to real-world applications in biomedical research and diagnostics.

\section{Acknowledgments}

We thank Vikas Kumar and Giulio Cerullo for fruitful discussions. We furthermore gratefully acknowledge financial support by the ERC Advanced Grant (COMPLEXPLAS); DFG (Nos. SPP1391, SPP 1839, FOR730, and GI 269/11-1); the Bundesministerium für Bildung und Forschung (Nos. 13N9048, 13N10146, and PRINTOPTICS), the Carl Zeiss Foundation; the Baden-Württemberg Stiftung (Spitzenforschung II); the University of Stuttgart (open access fund); the EPSRC (No. EP/P001114/1); and the SRPe PRER and PECRE Award 2017/18.

\section{References}

1. W. Min et al., "Coherent nonlinear optical imaging: beyond fluorescence microscopy," Annu. Rev. Phys. Chem. 62, 507-530 (2011).

2. A. Zumbusch, W. Langbein, and P. Borri, "Nonlinear vibrational microscopy applied to lipid biology," Progr. Lipid Res. 52, 615-632 (2013).

3. Y. Yu, P. V. Ramachandran, and M. C. Wang, "Shedding new light on lipid functions with CARS and SRS microscopy," Biochim. Biophys. Acta 1841, 1120-1129 (2014).

4. F.-K. Lu et al., "Label-free DNA imaging in vivo with stimulated Raman scattering microscopy," Proc. Natl. Acad. Sci. U. S. A. 112, 11624-11629 (2015).

5. M. Cicerone, "Molecular imaging with CARS micro-spectroscopy," Curr. Opin. Chem. Biol. 33, 179-185 (2016).

6. C. H. Camp and M. T. Cicerone, "Chemically sensitive bioimaging with coherent Raman scattering," Nat. Photonics 9, 295-305 (2015).

7. F. Masia et al., "Quantitative chemical imaging and unsupervised analysis using hyperspectral coherent anti-Stokes Raman scattering microscopy," Anal. Chem. 85, 10820-10828 (2013).

8. C. W. Freudiger et al., "Label-free biomedical imaging with high sensitivity by stimulated Raman scattering microscopy," Science 322, 1857-1861 (2008).

9. P. Nandakumar, A. Kovalev, and A. Volkmer, "Vibrational imaging based on stimulated Raman scattering microscopy," New J. Phys. 11, 033026 (2009).

10. A. Dogariu et al., "Concentration dependence in coherent Raman scattering," J. Mod. Opt. 55, 3255-3261 (2008).

11. Y. Ozeki et al., "Analysis and experimental assessment of the sensitivity of stimulated Raman scattering microscopy," Opt. Express 17, 3651-3658 (2009).

12. J. Réhault et al., "Broadband stimulated Raman scattering with Fourier-transform detection," Opt. Express 23, 25235-25246 (2015).

13. W. Langbein, I. Rocha-Mendoza, and P. Borri, "Coherent antiStokes Raman micro-spectroscopy using spectral focusing: theory and experiment," J. Raman Spectrosc. 40, 800-808 (2009).

14. I. Rocha-Mendoza et al., "Differential coherent anti-Stokes Raman scattering microscopy with linearly chirped femtosecond laser pulses," Opt. Lett. 34, 2258-2260 (2009).

15. F. Saltarelli et al., "Broadband stimulated Raman scattering spectroscopy by a photonic time stretcher," Opt. Express 24, 21264-21275 (2016).

16. K. Tokunaga et al., "Generation of synchronized picosecond pulses by a $1.06-\mu \mathrm{m}$ gain-switched laser diode for stimulated Raman scattering microscopy," Opt. Express 24, 9617-9628 (2016).

17. S. Karpf et al., "A time-encoded technique for fibre-based hyperspectral broadband stimulated Raman microscopy," Nat. Commun. 6, 6784 (2015).

18. C. W. Freudiger et al., "Stimulated Raman scattering microscopy with a robust fibre laser source," Nat. Photonics 8, 153-159 (2014).

19. C. Riek et al., "Stimulated Raman scattering microscopy by Nyquist modulation of a two-branch ultrafast fiber source," Opt. Lett. 41, 3731-3734 (2016).

20. D. A. Orringer et al., "Rapid intraoperative histology of unprocessed surgical specimens via fibre-laser-based stimulated Raman scattering microscopy," Nat. Biomed. Eng. 1, 0027 (2017). 
21. T. Südmeyer et al., "Novel ultrafast parametric systems: high repetition rate single-pass OPG and fibre-feedback OPO," J. Phys. D 34, 2433-2439 (2001).

22. F. Mörz et al., "Multi-Watt femtosecond optical parametric master oscillator power amplifier at $43 \mathrm{MHz}$," Opt. Express $\mathbf{2 3}$, 23960-23967 (2015).

23. M. Marangoni et al., "Narrow-bandwidth picosecond pulses by spectral compression of femtosecond pulses in a second-order nonlinear crystal," Opt. Express 15, 8884-8891 (2007).
24. Q. Hu, H. Zhao, and S. Ouyang, "Understanding water structure from Raman spectra of isotopic substitution $\mathrm{H}_{2} \mathrm{O} / \mathrm{D}_{2} \mathrm{O}$ up to 573 K," Phys. Chem. Chem. Phys. 19, 21540-21547 (2017).

25. T. W. Kee and M. T. Cicerone, "Simple approach to one-laser, broadband coherent anti-Stokes Raman scattering microscopy," Opt. Lett. 29, 2701-2703 (2004).

Biographies of the authors are not available. 